\title{
Risk Assessment and Management of File Server Components in Production Activities
}

\author{
Chernyshova E.V.* \\ Department of Information Security \\ Voronezh State University of Engineering Technologies \\ Voronezh, Russia \\ e-mail: elenabok@mail.ru \\ Razgonyeva V.V. \\ Department of Information Security \\ Voronezh State University of Engineering Technologies \\ Voronezh, Russia \\ Zelikov V.A. \\ Department of Information Security \\ Voronezh State University of Engineering Technologies \\ Voronezh, Russia
}

\author{
Strukov Y.V. \\ Department of Information Security \\ Voronezh State University of Engineering Technologies \\ Voronezh, Russia \\ Maklakova E.A. \\ Department of Information Security \\ Voronezh State University of Engineering Technologies \\ Voronezh, Russia \\ Skrypnikov A.A. \\ Department of Information Security \\ Voronezh State University of Engineering Technologies \\ Voronezh, Russia
}

\begin{abstract}
The paper is devoted to mathematical modeling of the analysis of the survival of an automated information system in enterprises, namely the survival of a file server for production management. Based on the studies, a methodology was developed for constructing a survival analysis model for an automated information system, which consisted of using beta distributions as a survival analysis model. This model can effectively predict the behavior of a system after an attack and the prospective life expectancy of both the whole system and its separate components, calculating risk parameters for a beta distribution of the probability density of damage and building the dependence of the values of the estimates of the survival function and instant risk function on specific factors.
\end{abstract}

Keywords - file server, data protection, beta distribution, risk assessment.

\section{INTRODUCTION}

When analyzing the survival of an automated information system (AIS), we use the sensitivity function and construct a sensitivity matrix. The sensitivity function is used in two ways:

$$
\begin{aligned}
& \text { differential sensitivity: } S=\frac{\partial X}{\partial A} \\
& \text { relative sensitivity: } S_{A}^{X}=\frac{\partial(\ln X)}{\partial(\ln A)}=\frac{\partial X / X}{\partial A / A}
\end{aligned}
$$

The selected set of parameters $\bar{A}$, which we will call the base, corresponds to the set of state variables. Let the set of input and output state variables be the set $\mathrm{Y}$. We will consider this set.
The dependence of variables on time and the set of parameters is expressed by the formula:

$$
\overline{y_{l}}=y_{i}(t, \bar{A})
$$

which is called the primary or basic movement.

The vector $\Delta y_{i}$ defined by the relation: $\Delta y_{i}=y_{i}(t, \bar{A}+$ $\Delta A)-y_{i}(t, \bar{A})$

is called the additional movement caused by a change in the influence of the parameters A. An additional movement characterizes the change in the properties of the information system, which is of interest to the researcher when the parameters change. Therefore, the study of the properties of additional movements and the establishment of their relationship with the properties of the original information system is the main task of sensitivity research.

We establish the connection between the sensitivity functions and the additional movement. To do this, we use the properties of higher order differentials of functions of several variables. For example, $Y=Y\left(x_{1}, \ldots, x_{r}\right)$ is a function of variables. $x_{1}, \ldots, x_{r}$. Then $\mathrm{k}$-th order differentials are called the expressions:

$$
d^{(k)} Y=\left(\frac{\partial}{\partial x_{1}} d x_{1}+\ldots+\frac{\partial}{\partial x_{r}} d x_{r}\right)^{k} Y,
$$

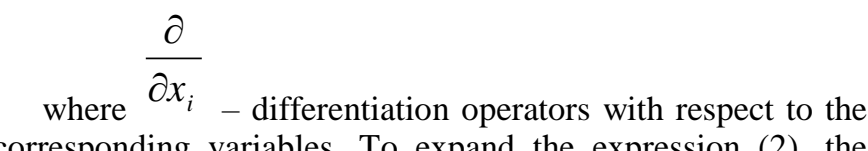


expression in parentheses should be formally raised to a power, and the resulting operator should be applied to the function Y. By the Taylor formula with the remainder term, we obtain:

$$
\begin{gathered}
\Delta Y=Y(t, \bar{A}+\Delta A)-Y(t, \bar{A})=d Y(t, \bar{A})+\frac{1}{2 !} d^{(2)} Y(t, \bar{A})+\ldots+ \\
+\frac{1}{n !} d^{(n)} Y(t, \bar{A})+\frac{1}{(n+1) !} d^{(n+1)} Y(t, \bar{A}+\theta \Delta A)
\end{gathered}
$$

In the formula (3) the variable $t$ is considered to be an unchanged parameter. Neglecting the remainder term from (3), we obtain an approximate expression for the additional movement

$$
\Delta^{(k)} Y(t, A)=\left.\sum_{i=1}^{k} \frac{1}{i !} d^{(i)} Y(t, A)\right|_{A=\bar{A}} \text {. }
$$

which is called the $\mathrm{k}$-th approximation for additional movement. The value:

$$
\Delta_{k} Y=\frac{1}{k !} d^{(k)} Y \text {. }
$$
called the correction of the k-th order of additional movement.

Taking into account (4) and (2), the k-th order correction is determined by the relation:

$$
\Delta_{k} Y=\left.\frac{1}{k !}\left(\frac{\partial}{\partial \alpha_{1}} \Delta \alpha_{1}+\ldots+\frac{\partial}{\partial \alpha_{r}} \Delta \alpha_{r}\right)^{k} Y(t, \alpha)\right|_{A=\bar{A}} .
$$

It follows that the correction of the $\mathrm{k}$-th order is a linear form of the sensitivity functions of the kth order calculated for the basic values of the parameters, as well as the form of the k-th order with respect to the increments of the parameters $\Delta \alpha_{1}, \ldots, \Delta \alpha_{r}$.

Corresponding formulas can also be obtained for quality indicators $K_{i}$, corresponding to variables $y_{i}$.

Having a set of sensitivity functions for an arbitrary complete set of parameters $A$, you we can get $\Delta Y(t, A) \approx \Delta^{(1)} Y(t, A)$

using an approximate representation of the additional motion (7) and

$$
\Delta \alpha_{i}=\sum_{j=1}^{q} \frac{\partial \alpha_{i}}{\partial \beta_{j}} \Delta \beta_{j}
$$

increment (8) and study the effect on the additional movement of arbitrary parameters associated with $A$ by relations (6)

Unlike the first approximation, higher-order approximations are invariant with respect to transformation (8) only if this transformation is linear. If the approximate equality is fulfilled with sufficient accuracy, then we can simply analyze the various properties of the additional motion. Let us calculate the risk sensitivity for the parameter $\alpha$ :

$$
\begin{gathered}
\frac{\partial B(\alpha, \beta)}{\partial \alpha}=\frac{\partial}{\partial \alpha} \int_{0}^{1} x^{\alpha-1}(1-x)^{\beta-1} d x=(\alpha-1) \int_{0}^{1} x^{\alpha-2}(1- \\
x)^{\beta-1} d x=(\alpha-1) B(\alpha-1, \beta) \\
\frac{\partial}{\partial \alpha}\left(\frac{B(\alpha, \beta)\left(\frac{\alpha+\beta-2}{\beta-1}\right)^{\alpha+\beta-2}}{n\left(\frac{\alpha-1}{\beta-1}\right)^{\alpha-1}}\right)=\frac{\left(\frac{\frac{\alpha^{2}}{2}+\beta \alpha-2 \alpha}{(\beta-1)^{2}}\right)^{\alpha+\beta-1} n\left(\frac{\alpha+\beta-2}{\beta-1}\right)^{\alpha+\beta-2}\left(\frac{\alpha-1}{\beta-1}\right)^{\alpha-1}}{n\left(\frac{\alpha-1}{\beta-1}\right)^{2 \alpha-2}}- \\
-\frac{n\left(\frac{\alpha-1}{\beta-1}\right)^{\alpha-1}\left(\frac{\frac{\alpha^{2}}{2}-\alpha}{(\beta-1)^{2}}\right)^{\alpha}\left(\frac{\alpha+\beta-2}{\beta-1}\right)}{n\left(\frac{\alpha-1}{\beta-1}\right)^{2 \alpha-2}}, \\
\frac{(\Delta t)^{\prime} t^{\alpha-1} B(\alpha, \beta)-t^{\alpha-1} \Delta t[(\alpha-1) B(\alpha-1, \beta)+B(\alpha, \beta) \ln (1+t)]}{B^{2}(\alpha, \beta)(1+t)^{\alpha+\beta-2}}
\end{gathered}
$$

We calculate the risk sensitivity for the parameter $\beta \beta$ :

$$
\begin{aligned}
& \frac{\partial B(\alpha, \beta)}{\partial \beta}=\frac{\partial}{\partial \beta} \int_{0}^{1} x^{\alpha-1}(1-x)^{\beta-1} d x \\
= & (\beta-1) \int_{0}^{1} x^{\alpha-1}(1-x)^{\beta-2} d x \\
= & (\beta-1) B(\alpha, \beta-1)
\end{aligned}
$$

$$
\frac{\partial \Delta t}{\partial \beta}=
$$

$$
\begin{aligned}
& \frac{\partial}{\partial \beta}\left(\frac{B(\alpha, \beta)\left(\frac{\alpha+\beta-2}{\beta-1}\right)^{\alpha+\beta-2}}{n\left(\frac{\alpha-1}{\beta-1}\right)^{\alpha-1}}\right)=\frac{\left(\frac{\frac{\beta^{2}}{2}+\beta \alpha-2 \beta}{(\beta-1)^{2}}\right)^{\alpha+\beta-1} n\left(\frac{\alpha+\beta-2}{\beta-1}\right)^{\alpha+\beta-2}\left(\frac{\alpha-1}{\beta-1}\right)^{\alpha-1}}{n\left(\frac{\alpha-1}{\beta-1}\right)^{2 \alpha-2}}- \\
& -\frac{\left(\frac{\alpha-1}{\beta-1}\right)^{\alpha-1}\left(\frac{\frac{\beta^{2}}{2}-\beta}{(\beta-1)^{2}}\right)^{\alpha}\left(\frac{\alpha+\beta-2}{\beta-1}\right)}{n\left(\frac{\alpha-1}{\beta-1}\right)^{2 \alpha-2}},
\end{aligned}
$$

$S_{\beta}=\frac{\partial R i s k}{\partial \beta}=$

$\frac{(\Delta t)^{\prime} t^{\beta-1} B(\alpha, \beta)-t^{\beta-1} \Delta t[(\beta-1) B(\alpha, \beta-1)+B(\alpha, \beta) \ln (1+t)]}{B^{2}(\alpha, \beta)(1+t)^{\alpha+\beta-2}}$

We find the relative sensitivity:

$S_{\alpha}^{l n}$ Risk $=$ $\frac{(\Delta t)^{\prime} t_{0}{ }^{\alpha-1} B(\alpha, \beta)-t_{0}{ }^{\alpha-1} \Delta t\left[(\alpha-1) B(\alpha-1, \beta)+B(\alpha, \beta) \ln \left(1+t_{0}\right)\right]}{B(\alpha, \beta) \Delta t * \tau_{3}\left[B y\left(0, \frac{1}{\beta_{3}}+1\right)-B\left(0, \frac{1}{\beta_{3}}+1\right)\right] t_{0}{ }^{\alpha-1}}$

$$
\begin{gathered}
S_{\beta}^{l n} \text { Risk }= \\
\frac{(\Delta t)^{\prime} t^{\beta-1} B(\alpha, \beta)-t^{\beta-1} \Delta t[(\beta-1) B(\alpha, \beta-1)+B(\alpha, \beta) \ln (1+t)]}{B(\alpha, \beta) \Delta t * \tau_{3}\left[B y\left(0, \frac{1}{\beta_{3}}+1\right)-B\left(0, \frac{1}{\beta_{3}}+1\right)\right] t_{0}{ }^{\alpha-1}}
\end{gathered}
$$

Having obtained the relative sensitivity coefficients, we can construct a sensitivity matrix. In general, the sensitivity matrix is as follows:

$$
S^{\text {Risk }}=\left[\begin{array}{ccc}
S_{x_{i}}^{\text {Risk(1) }} & S_{x_{i}}^{\text {Risk }(2)} & \cdots \\
S_{x_{i+1}}^{\text {Risk(1) }} & S_{x_{i+1}}^{\text {Risk(2) }} & \ldots \\
\cdots & \cdots & \ldots
\end{array}\right]
$$


We write the differential sensitivity matrix:

$S^{\text {Risk }}=\left[\begin{array}{l}\frac{(\Delta t)^{\prime} t^{\alpha-1} B(\alpha, \beta)-t^{\alpha-1} \Delta t[(\alpha-1) B(\alpha-1, \beta)+B(\alpha, \beta) \ln (1+t)]}{B^{2}(\alpha, \beta)(1+t)^{\alpha+\beta-2}} \\ \frac{(\Delta t)^{\prime} t^{\beta-1} B(\alpha, \beta)-t^{\beta-1} \Delta t[(\beta-1) B(\alpha, \beta-1)+B(\alpha, \beta) \ln (1+t)]}{B^{2}(\alpha, \beta)(1+t)^{\alpha+\beta-2}}\end{array}\right]$

We write the relative sensitivity matrix:

$S^{\ln R i s k}=$

$\left[\begin{array}{c}\frac{(\Delta t)^{\prime} t_{0}{ }^{\alpha-1} B(\alpha, \beta)-t_{0}{ }^{\alpha-1} \Delta t\left[(\alpha-1) B(\alpha-1, \beta)+B(\alpha, \beta) \ln \left(1+t_{0}\right)\right]}{B(\alpha, \beta) \Delta t * \tau_{3}\left[B y\left(0, \frac{1}{\beta_{3}}+1\right)-B\left(0, \frac{1}{\beta_{3}}+1\right)\right] t_{0}{ }^{\alpha-1}} \\ \frac{(\Delta t)^{\prime} t^{\beta-1} B(\alpha, \beta)-t^{\beta-1} \Delta t[(\beta-1) B(\alpha, \beta-1)+B(\alpha, \beta) \ln (1+t)]}{B(\alpha, \beta) \Delta t * \tau_{3}\left[B y\left(0, \frac{1}{\beta_{3}}+1\right)-B\left(0, \frac{1}{\beta_{3}}+1\right)\right] t_{0}{ }^{\alpha-1}}\end{array}\right]$

We study the behavior of the sensitivity function at various parameter values. To do this, we build the graphs (Fig. $01 \mathrm{a}-\mathrm{f}$ ).

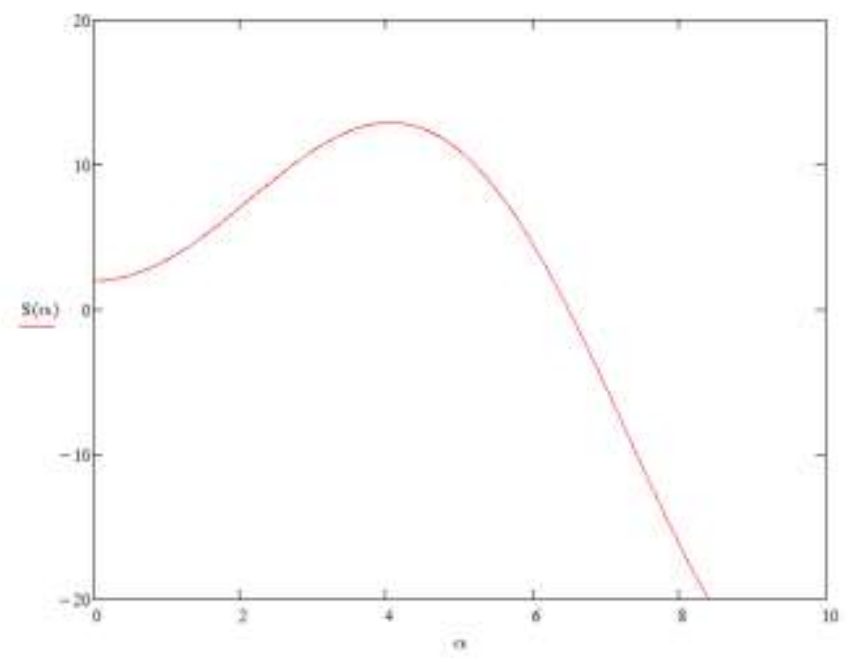

a) Differential sensitivity with respect to $\alpha$ at $\beta=2$

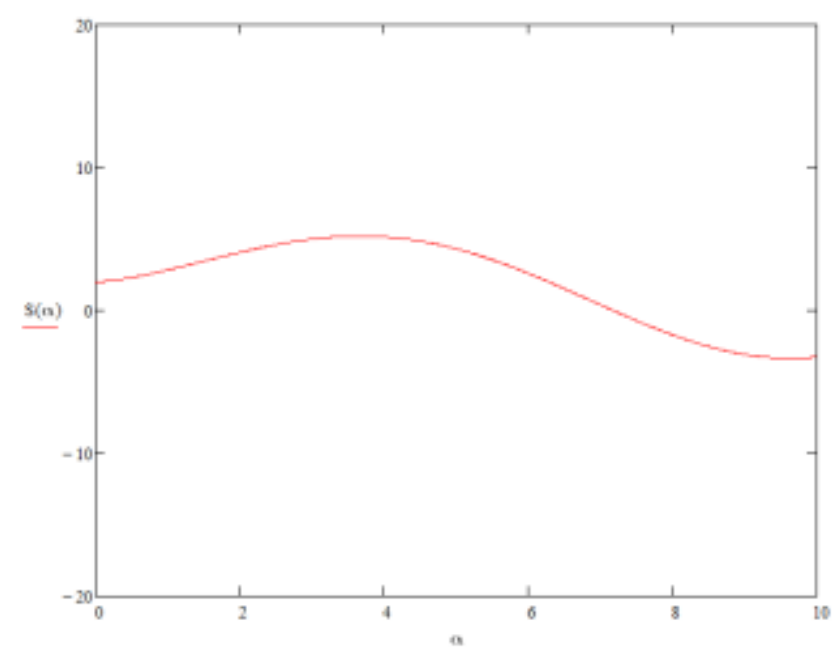

b) Differential sensitivity with respect to $\alpha$ at $\beta=1$

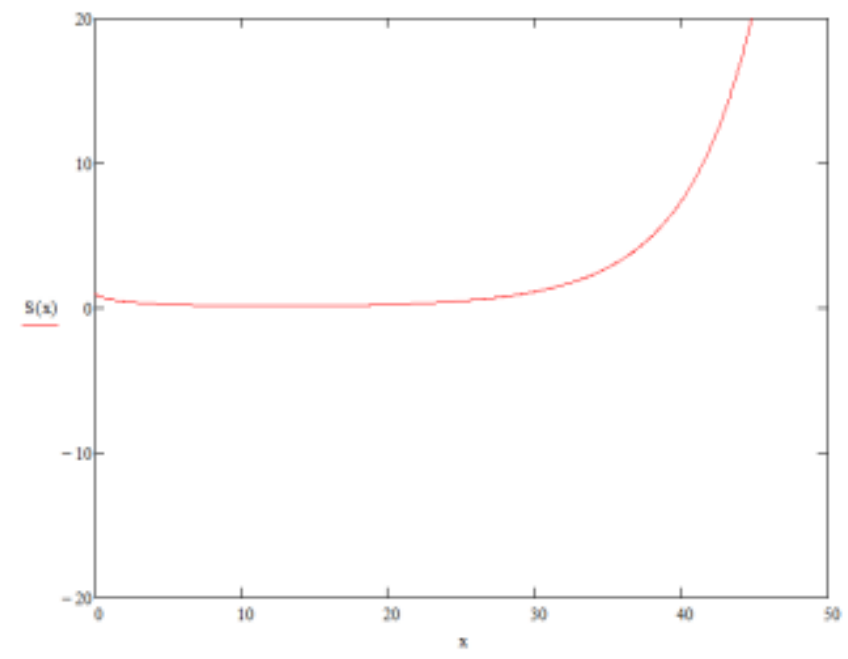

c) Control surface in parameter $\beta$

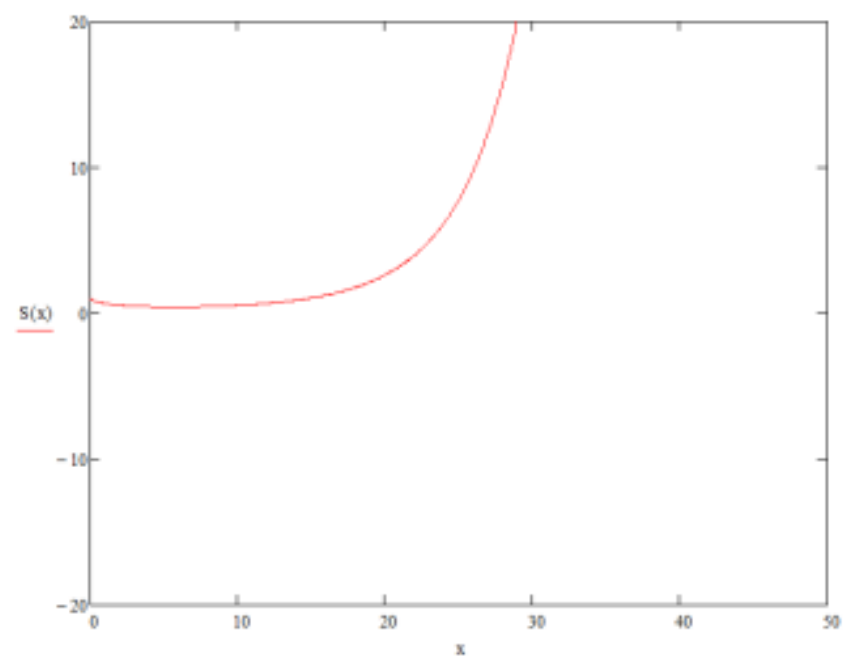

d) Control surface in parameter $\alpha$

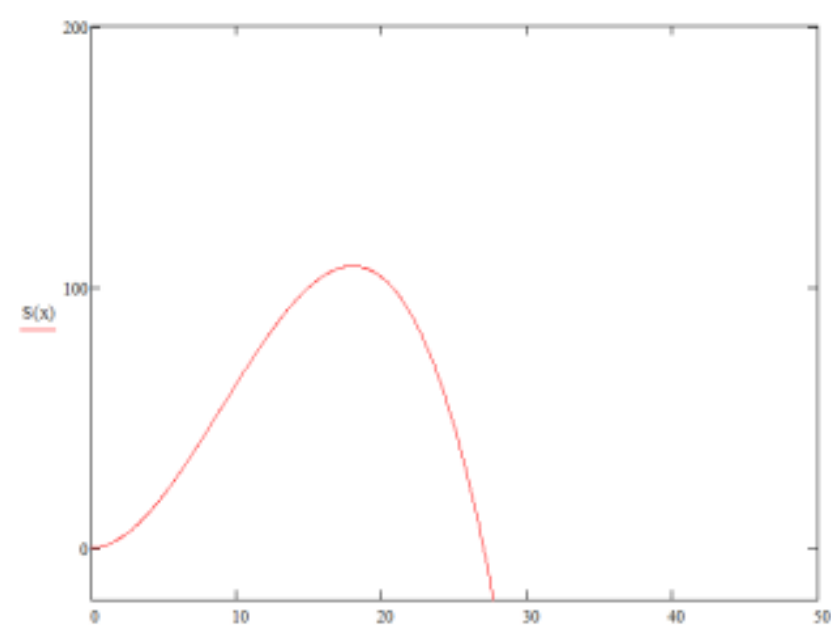

e) Relative sensitivity in parameter $\alpha$ 


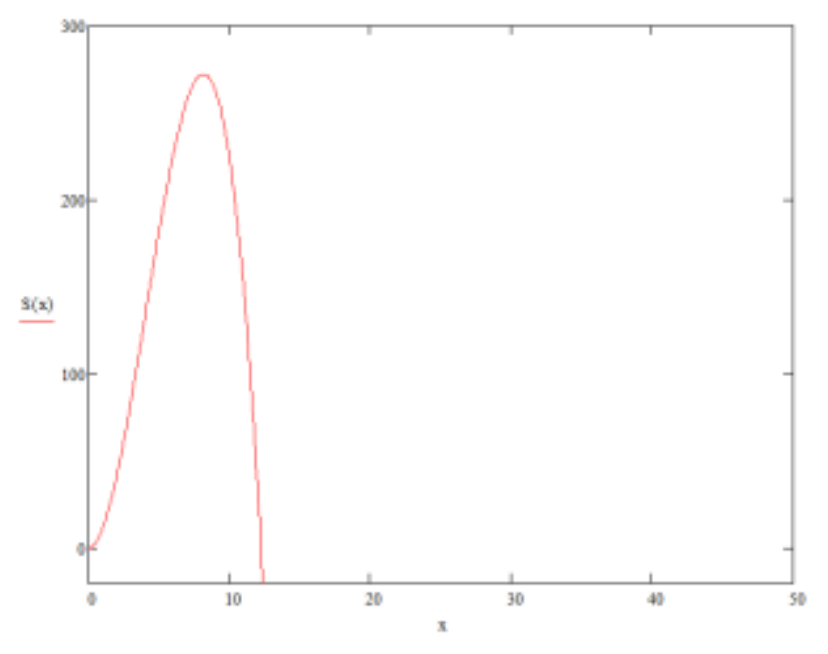

f) Relative sensitivity in parameter $\beta$

Figure 01. Sensitivity function at various parameter values

\section{CONCLUSIONS}

The graphs show the behavior of sensitivity at various parameters and allow a general risk assessment for the file server. The studies performed in the paper make it possible to assess the dynamic behavior of risk over time when working on a file server, which is necessary for choosing a risk management strategy and its direct management in production activities.
The graphs showed the behavior of sensitivity at various parameters and allow a general risk assessment for the file server. The research allowed assessing the dynamic behavior of risk over time when working on a file server. This result is necessary for choosing a risk management strategy and its direct management in production activities.

\section{References}

[1] A.V. Skrypnikov, S.V. Dorokhin, V.G. Kozlov and E.V. Chernyshova, "Mathematical Model of Statistical Identification of Car Transport Informational Provision," Journal of Engineering and Applied Sciences, Vol. 12-2, pp. 511-515, January 2017.

[2] A.V. Skrypnikov, V.G. Kozlov, A.N. Belyaev, E.V. Chernyshova, "Theoretical foundations of the method of designing a clothoid track with approximation of succession of points," Advances in Intelligent Systems and Computing. 2019, vol. 726, pp. 654-667.

[3] V.G. Kozlov, A.V. Skrypnikov, E.V. Chernyshova, R.V. Mogutnov, D.M. Levushkin "Mathematical modeling of damage function when attacking file server," Journal of Physics: Conference Series, 2018. vol. 1015. p. 032069.

[4] V.G. Kozlov, S.I. Sushkov, I.N. Kruchinin, I.V. Grigorev, "Enhancing quality of road pavements through adhesion improvement," Journal of the Balkan Tribological Association, 2019, vol. 25-3, pp. 678-694.

[5] Y.A. Safonova, M.N. Ivliev, A.V. Lemeshkin, "Chickpea seeds germination rational parameters optimization," Journal of Physics: Conference Series, 2018, vol. 1015, p. 032118.

[6] S.V. Belokurov, "Construction of invariant functions of choice and study of probabilistic characteristics for binary relations on the pareto set," Automation and Remote Control, 2011, vol. 72-11, p. 2395-2401.

[7] P.S. Ptitsyn, D.V. Radko, O.V. Lankin, "Designing architecture of software framework for building security infrastructure of global distributed computing systems," ARPN Journal of Engineering and Applied Sciences, 2016. vol. 11-19, p. 11599-11610. 